\title{
Pheochromocytoma Mimicking both Acute Coronary Syndrome and Sepsis: A Case Report
}

\author{
Ting-Wei Lee ${ }^{a} \quad$ Ke-Hsun Lin ${ }^{b} \quad$ Chun-Jen Chang ${ }^{a} \quad$ Wei-Han Lew ${ }^{a}$ Ting-I Lee ${ }^{a}$ c \\ ${ }^{a}$ Division of Endocrinology and Metabolism, Department of Internal Medicine, and ${ }^{\mathrm{b}}$ Department of Urology, \\ Wan Fang Hospital, Taipei Medical University, and ${ }^{C}$ Department of General Medicine, School of Medicine, College \\ of Medicine, Taipei Medical University, Taipei, Taiwan
}

\section{Key Words}

Pheochromocytoma - Coronary artery disease - Sepsis .

Catecholamine

\begin{abstract}
Objective: To present an atypical manifestation in a patient with pheochromocytoma. Clinical Presentation and Intervention: $\mathrm{A}$ 48-year-old man presented with chest pain, fever and leukocytosis. Elevated cardiac biomarkers and diffuse ST-T abnormalities on electrocardiography suggested myocardial infarction. However, coronary angiography showed normal coronary arteries. Abdominal computed tomography revealed a left adrenal tumor of $6.7 \times 6.8 \mathrm{~cm}$. Paroxysmal fluctuation of blood pressure raised the suspicion of pheochromocytoma, which was further supported by elevated urine catecholamine levels. He underwent left adrenalectomy and pathological findings confirmed the diagnosis. Conclusion: Pheochromocytoma should be considered as part of the differential diagnosis in a patient with symptoms suggestive of both acute coronary syndrome and sepsis.
\end{abstract}

\section{Introduction}

Pheochromocytoma is a rare catecholamine-producing neuroendocrine tumor originating in the chromaffin cells of the adrenal medulla or extra-adrenal paraganglia $[1,2]$. Classically, it presents with paroxysmal or sustained hypertension accompanied by episodic symptoms of headache, palpitation, and diaphoresis $[1,2]$. Herein, we report a patient presenting with symptoms suggestive of acute coronary syndrome (ACS) and sepsis that was later diagnosed as pheochromocytoma.

\section{Case Report}

A 48-year-old man presented with acute onset of substernal chest pain radiating to the back. He had a history of hypertension and hyperlipidemia with inconsistent follow up for three years. He denied having paroxysmal symptoms of headache, palpitation, or diaphoresis. On examination, the patient's body temperature was $39.9^{\circ} \mathrm{C}$, blood pressure (BP) $157 / 87 \mathrm{~mm} \mathrm{Hg}$, and pulse 92 $\mathrm{bpm}$. Abnormal laboratory results were as follows: WBC $23.52 \times$ $10^{9} / 1$ with $88 \%$ neutrophils, C-reactive protein $7.1 \mathrm{mg} / \mathrm{l}$ (normal $0-5 \mathrm{mg} / \mathrm{l}$ ), and troponin-I $4.3 \mathrm{ng} / \mathrm{ml}$ (normal 0-0.5 ng/ml). Electrocardiography (ECG) revealed no significant ST-T abnormalities (fig. 1a). Transthoracic echocardiogram showed dilated left ventricular (LV) chamber without regional wall motion abnormality, and LV systolic contractility was preserved. A non-ST elevation myocardial infarction was highly suspected. However,

Ting-I Lee, MD, PhD

Wan Fang Hospital, Taipei Medical University

No. 111, Section 3, Xinglong Road

Taipei 11696, Taiwan (ROC)

E-Mail aglee@ms29.hinet.net Creative Commons Attribution-NonCommercial 3.0 Unported license (CC BY-NC) (www.karger.com/OA-license), applicable to the online version of the article only. Distribution permitted for non-commercial purposes only. 
Fig. 1. Electrocardiograms. a No ST-T abnormalities. b Diffuse ST segment depression.
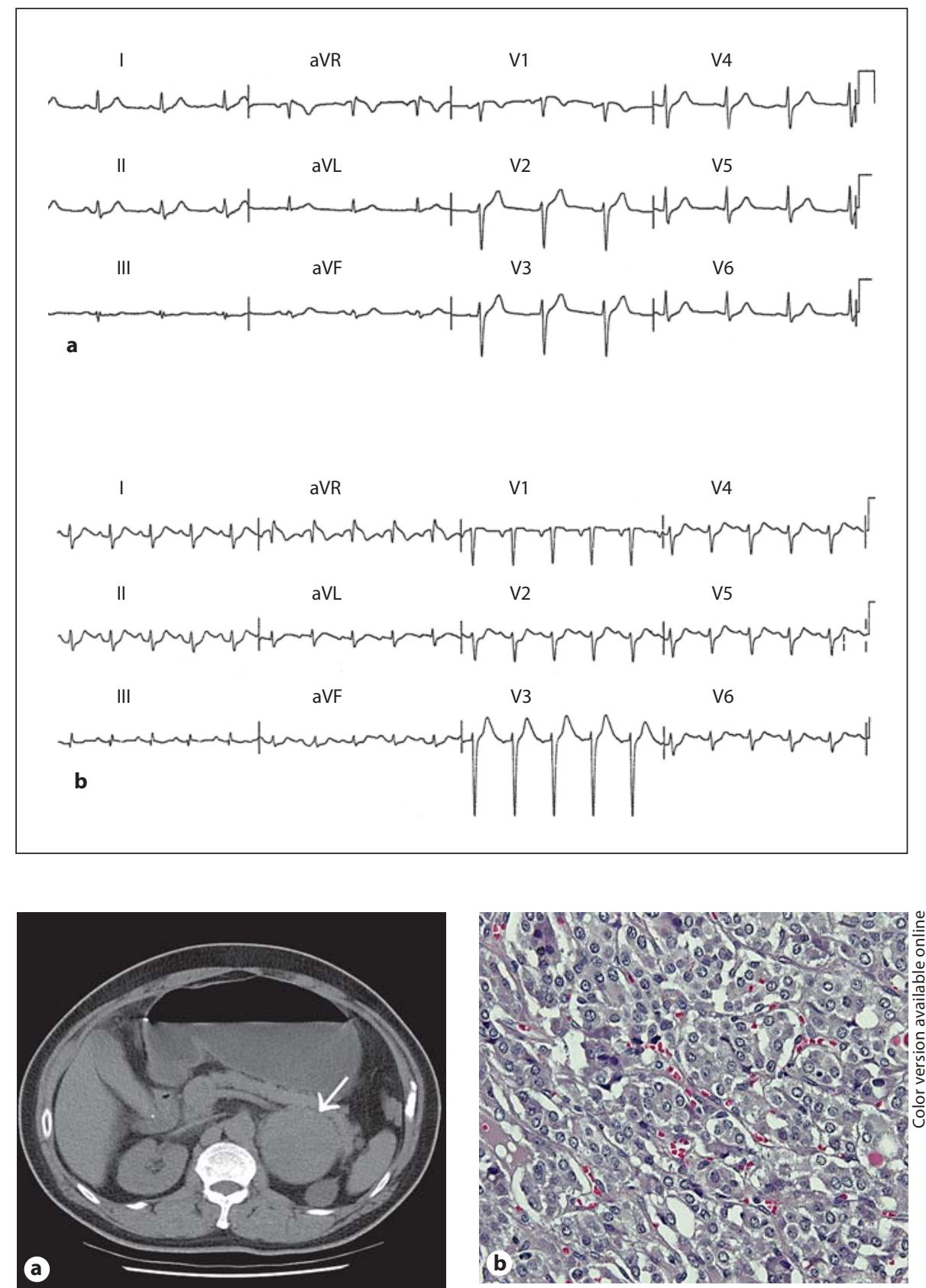

Fig. 2. a Abdominal computed tomography showed a well-defined tumor about $6.7 \times 6.8 \mathrm{~cm}$ at the left adrenal gland without calcification. b Pheochromocytoma cells stained with hematoxylin and eosin stain, at $\times 400$ magnification under a microscope. The tumor cells had small nuclei and abundant finely granular amphophilic cytoplasm and were arranged in solid nests and organoid patterns. coronary angiography revealed normal coronary arteries. There was no BP fluctuation during his first hospitalization. He became afebrile and leukocytosis remitted on the second hospital day without antibiotic treatment. Blood and urine cultures were all negative for microbiological pathogens.

Four months later, he was readmitted due to recurrent episode of high-grade fever and acute onset of substernal chest pain. His troponin-I $9.5 \mathrm{ng} / \mathrm{ml}$ was again highly elevated. ECG this time showed diffuse ST segment depression (fig. 1b). However, the negative finding of his last coronary angiography made myocardial infarction type 1 unlikely. Other results of blood test were: WBC
$34.18 \times 10^{9} / 1$ with $90.5 \%$ neutrophils, C-reactive protein $58.3 \mathrm{mg} / \mathrm{l}$, aspartate transaminase $145 \mathrm{U} / \mathrm{l}$ (normal 5-30 U/l), alanine transaminase $110 \mathrm{U} / \mathrm{l}$ (normal 5-35 U/1). Abdominal computed tomography, which was performed to search for the infection source, revealed a well-defined tumor about $6.7 \times 6.8 \mathrm{~cm}$ at the left adrenal gland (fig. 2a). During the second hospital course, his BP fluctuated from 92/81 to 191/101 mm Hg. The apparent ACS and sepsis, labile BP, associated with left adrenal tumor raised the suspicion of pheochromocytoma, which was further supported by markedly elevated 24-hour urine levels of catecholamine: epinephrine 590.2 $\mu \mathrm{g} /$ day (normal $<27 \mu \mathrm{g} /$ day), norepinephrine $755.5 \mu \mathrm{g} /$ day (nor- 
mal $<97 \mu \mathrm{g} /$ day), dopamine $821.7 \mu \mathrm{g} /$ day (normal $<500 \mu \mathrm{g} /$ day) and vanillylmandelic acid (VMA): $114.2 \mathrm{mg} /$ day (normal 1.0-7.5 $\mathrm{mg} /$ day). Ceftriaxone was administered for one week because of high suspicion of sepsis. On the third hospital day, he became afebrile and leukocytosis resolved. Culture survey for infectious pathogens was negative. He underwent left laparoscopic adrenalectomy, and the pathological findings confirmed our diagnosis (fig. 2b). Postoperatively, the patient remained asymptomatic with normal urine levels of catecholamine and VMA for 3 years.

\section{Discussion}

Pheochromocytoma can present with a multitude of symptoms mimicking other diseases. Yu et al. [3] had reported that patients with pheochromocytoma initially present with cardiac complications, such as MI, heart failure, and arrhythmia, often delaying a diagnosis of pheochromocytoma. Because of normal coronary arteries on angiography, the patient's chest pain, associated with markedly elevated cardiac biomarkers and diffuse ST segment abnormalities on ECG, an initial diagnosis of MI type 2 was made. However, fluctuations of BP during his second hospitalization provided us a hint of catecholamine excess. The pathogenesis of pheochromocytomarelated cardiac complications is thought to be a cardiotoxic effect of catecholamine. Substantial evidence exists to indicate that coronary spasm, alterations in lipid and energy metabolism, changes in cell membrane permeability, and intracellular calcium overload contribute to catecholamine-induced cardiotoxicity $[4,5]$. In addition, oxidative stress and oxidation of catecholamines have been associated with cardiac injury, both in vitro and in vivo studies $[6,7]$. To reach a timely diagnosis of the car- diovascular presentations of pheochromocytoma, physicians should expand the differential diagnosis to include rarer diseases if the initial cardiac workup did not reveal a clear etiology.

The causes of fever in pheochromocytoma are multifactorial and often include an associated illness [8-10]. In addition to hypermetabolism and impaired heat dissipation, interleukin- 6 released by pheochromocytoma is also relevant to fever [9]. Our patient presented with symptoms suggestive of both ACS and sepsis, which has rarely been described in the literature review of pheochromocytoma. In the reported cases, a focus of infection was identified and the findings of sepsis and cardiac injury persisted [10]. Our patient was unusual in that he had no associated infection. Since the combination of apparent ACS and sepsis has been reported with an infection in a patient with pheochromocytoma, empiric antibiotics should be considered pending culture results if this presentation is encountered.

\section{Conclusion}

This case showed that pheochromocytoma must be considered as part of the differential diagnosis in a patient presenting with symptoms suggestive of both acute coronary syndrome and sepsis.

\section{Acknowledgement}

The present work was supported by grant 101-wf-eva-02 from Taipei Medical University-Wan Fang Hospital.

\section{References}

-1 Erem C, Hacihasanoglu A, Cinel A, Isik AC, Reis A, Sari A, Ersoz HO, Ukinc K: Carotid body tumors and adrenal pheochromocytomas in siblings of a Turkish family. Med Princ Pract 2006;15:396-400.

-2 Reisch N, Peczkowska M, Januszewicz A, Neumann HP: Pheochromocytoma: presentation, diagnosis and treatment. J Hypertens 2006;24:2331-2339.

3 Yu R, Nissen NN, Bannykh SI: Cardiac complications as initial presentation of pheochromocytoma: frequency, outcome, and predictors. Endocr Pract 2012;31:1-27.
4 Behonick GS, Novak MJ, Nealley EW, Baskin SI: Toxicology update: the cardiotoxicity of the oxidative stress metabolites of catecholamines (aminochromes). J Appl Toxicol 2001;21(suppl 1):S15-S22.

5 Babick AP, Dhalla NS: Role of subcellular remodeling in cardiac dysfunction due to congestive heart failure. Med Princ Pract 2007; 16:81-89.

-6 Costa VM, Carvalho F, Bastos ML, Carvalho RA, Carvalho M, Remiao F: Contribution of catecholamine reactive intermediates and oxidative stress to the pathologic features of heart diseases. Curr Med Chem 2011;18:2272-2314.

77 Walia M, Kwan CY, Grover AK: Effects of free radicals on coronary artery. Med Princ Pract 2003;12:1-9.
$>8$ Gordon DL, Atamian SD, Brooks MH, Gattuso P, Castelli MJ, Valaitis J, Thomas W Jr: Fever in pheochromocytoma. Arch Intern Med 1992;152:1269-1272.

$>9$ Yarman S, Soyluk O, Altunoglu E, Tanakol R: Interleukin-6-producing pheochromocytoma presenting with fever of unknown origin. Clinics 2011;66:1843-1845.

10 Tanriver Y, Betz MJ, Nibbe L, Pfluger T, Beuschlein F, Strowski MZ: Sepsis and cardiomyopathy as rare clinical manifestations of pheochromocytoma - two case report studies. Exp Clin Endocrinol Diabetes 2010; 118:747-753.
Pheochromocytoma Mimicks Acute

Coronary Syndrome and Sepsis
Med Princ Pract 2013;22:405-407 DOI: $10.1159 / 000343578$ 